\title{
General Solution to a Bilinear Reduction of the Higher Order Nonlinear Schrödinger Equation
}

\author{
P.P. Goldstein* \\ Theoretical Physics Department \\ The Andrzej Soltan Institute for Nuclear Studies \\ Hoża 69, 00-681 Warsaw, Poland
}

(Received July 21, 2006)

\begin{abstract}
The general solution is found to a bilinear reduction of the higher order nonlinear Schrödinger equation. Except for the previously known special cases having multisoliton solutions, the solution has the form of a single envelope solitary wave or wave train for all other values of the parameters. We conjecture that the generic solution is limited to such a narrow class of functions because the bilinear system is overdetermined.
\end{abstract}

PACS numbers: 42.81.Dp

\section{Introduction}

It is a truth universally acknowledged that the closer an equation is to reality, the less it is likely to be integrable. On the other hand, even nonintegrable parameter-dependent equations have special values of their parameters for which the integrability has been established. For optical solitons one of the paths from integrable to realistic begins with the completely integrable nonlinear (cubic) Schrödinger equation (NLS) [1]

$$
\mathrm{i} A_{z}+A_{t t}+|A|^{2} A=0
$$

where $A$ is the slowly varying envelope of the electric field. NLS takes into account merely the group velocity dispersion (term $A_{t t}$ ), and the self-modulation of the phase (the nonlinear term). Further along the path we encounter equations of the type

$$
A_{z}=\mathrm{i}\left(A_{t t}+|A|^{2} A\right)+A_{t t t}+\gamma_{1}\left(|A|^{2} A\right)_{t}+\gamma_{2}\left(|A|^{2}\right)_{t} A,
$$

known as "higher order nonlinear Schrödinger equations" (HNLS or HONSE) [2-5]

*e-mail: Piotr.Goldstein@fuw.edu.pl 
which include also third-order dispersion (term $A_{t t t}$ ), self-steepening (Kerr dispersion - term $\gamma_{1}\left(|A|^{2} A\right)_{t}$ ), and frequency shifting via stimulated Raman scattering (term $\gamma_{2}\left(|A|^{2}\right)_{t} A$ ). Only special cases of Eq. (2) are integrable (see e.g. [2, 3]).

Existence of a solitary wave or even two-soliton solutions is not equivalent to integrability [6]. On the other hand, it is the elastic interaction of two solitons (and also stability of a soliton under small perturbations), which is most important for applications in communication.

The most effective search for soliton solutions is performed by the Hirota bilinear method $[7,8]$. By appropriate substitution the equations are cast in a bilinear form (see below), then the bilinear equations are solved by a perturbation technique (described in detail in [8]).

In Ref. [3] the authors look for solitary waves of the HONSE (2). First they do it by direct substitution of the single-soliton form

$$
A(z, t)=y(t+\beta z) \exp [\mathrm{i}(\kappa z-\Omega t)]
$$

and by identification of the integrable cases through the Painlevé test. Minute corrections to their work were presented in [9].

These authors also obtain the Hirota bilinear system of equations corresponding to (2). Substitution of $A=G / F$ into (2), where $G$ is complex while $F$ may be assumed real, yields an equation with extra degrees of freedom due to the introduction of two unknown functions instead of the single $A$. The authors of [3] make use of the extra degrees of freedom by following the usual procedure: they postulate separate equations for the linear part of (2). In this way they obtain the following Hirota-like system for the variables $G, F$ :

$$
\begin{gathered}
\left(D_{z}-\mathrm{i} D_{t}^{2}-D_{t}^{3}\right) G \cdot F=0, \\
(G \cdot F)\left[-\mathrm{i} D_{t}^{2}(F \cdot F)+\mathrm{i}\left(G^{*} \cdot G\right)+\left(\gamma_{1}+\gamma_{2}\right) D_{t}\left(G^{*} \cdot G\right)\right] \\
+D_{t}(G \cdot F)\left[-3 D_{t}^{2}(F \cdot F)+\left(3 \gamma_{1}+2 \gamma_{2}\right)\left(G^{*} \cdot G\right)\right]=0,
\end{gathered}
$$

where $D_{z}$ and $D_{t}$ are the Hirota bilinear differential operators defined by

$$
D_{z}^{m} D_{t}^{n} A \cdot B=\left.\left(\frac{\partial}{\partial z}-\frac{\partial}{\partial z^{\prime}}\right)^{m}\left(\frac{\partial}{\partial t}-\frac{\partial}{\partial t^{\prime}}\right)^{n} A(z, t) B\left(z^{\prime}, t^{\prime}\right)\right|_{z^{\prime}=z, t^{\prime}=t} .
$$

As we see, the 2 nd equation is not exactly bilinear in $G, G^{*}, F$ and this is a source of difficulties when they try the perturbation formalism. Failing to analyze Eqs. (4) directly, the authors of [3] do further reduction through the substitution

$$
G(z, t)=\bar{G}(z, t) \exp [\mathrm{i}(\kappa z-\Omega t)]
$$

and assume

$$
\Omega=1 / 3, \quad \gamma_{1}=3 .
$$

For this special case they obtain a solution in the form of a wave whose envelope has the shape of the modified Korteweg de Vries (MKdV) $N$-soliton solution.

In the present work we derive the general solution for $A=G / F$, where $G, F$ satisfy the system (4), and we do this without making any extra assumptions on the parameters. Instead of using the perturbational scheme we attain it by 
direct integration methods: change of variables and the method of characteristics. Our solution encompasses all the values of the parameters which have not been treated by the previous authors $[3-5,10]$. The result is surprisingly simple: with the exception of the previously known cases the general solution has the form of a single envelope solitary wave or a train of waves. We attribute this to the fact that the system (4), when regarded as a system of real equations with real unknowns, has more equations than unknown functions, i.e. it is overdetermined. We have encountered such overdetermined Hirota bilinear systems in our previous work $[11,12]$ and the consequences of the excess number of equations were similar.

\section{Solution}

Equations (4a, 4b) apparently constitute a system of two complex equations with two unknown functions. However, they actually are an overdetermined system of four real equations: the real and imaginary parts of two Eqs. (4), having three real unknowns: $\operatorname{Re} G, \operatorname{Im} G$, and $F$. The solution relies on separating the real and imaginary parts in each of these equations.

The real part of (4b) divided by $(G F)$ may be written as

$$
\frac{1}{2}\left[\ln \left(G^{*} G / F^{2}\right)\right]_{t}\left[-3 D_{t}^{2}(F \cdot F)+\left(3 \gamma_{1}+2 \gamma_{2}\right) G^{*} G\right]=0
$$

whence either $G^{*} G / F^{2}$ is independent of $t$ or

$$
D_{t}^{2}(F \cdot F)=\left[\gamma_{1}+(2 / 3) \gamma_{2}\right] G^{*} G
$$

The first possibility corresponds to a trivial constant-amplitude solution for $A$. The second one has the form of the Hirota bilinear equation for the cubic NLS.

The imaginary part of (4b) divided by $(G F)$ reads

$$
-D_{t}^{2}(F \cdot F)+G^{*} G-\mathrm{i}\left(\gamma_{1}+\gamma_{2}\right) D_{t}\left(G^{*} \cdot G\right)=0 .
$$

Please note that $D_{t}\left(G^{*} \cdot G\right)$ is purely imaginary, hence the factor i in front of the last term makes that term real.

If we consider the non-constant solution of (8), then the substitution of (9) into (10) yields an equation

$$
\mathrm{i}\left(\gamma_{1}+\gamma_{2}\right) D_{t}\left(G^{*} \cdot G\right)-\left[1-\gamma_{1}-(2 / 3) \gamma_{2}\right]\left(G^{*} G\right)=0 .
$$

We should bear in mind that $D_{t} G^{*} \cdot G$ in (11) is a Hirota bilinear expression rather than just a derivative and thus (11) is not an ordinary differential equation with the unknown function $G^{*} G$. The solution depends on the values of $\gamma_{1}, \gamma_{2}$. There are three cases

Case 1: $\quad \gamma_{1}=-\gamma_{2}=3$.

In this case Eq. (11) becomes an identity and thus Eqs. (4a,b) no more constitute an overdetermined system. These values of $\gamma_{1}, \gamma_{2}$ correspond to the well-known Hirota equation $[10,2,3]$ (please note that here Hirota's name refers to yet another achievement of that author, connected but not identical with his bilinear method). In that case the system has $N$-soliton solutions whose explicit form may be found in $[10,3]$. 
Case 2: $\quad \gamma_{1}=-\gamma_{2}, \quad \gamma_{1} \neq 3$.

In this case Eq. (11) has only a trivial solution $G^{*} G=0$.

Case 3: $\quad \gamma_{1} \neq-\gamma_{2}$.

In this case Eq. (11) may explicitly be solved. By substitution

$$
G=\bar{G} \exp (\mathrm{i} \theta), \quad \text { with } \bar{G}, \theta \text { real, }
$$

the equation may be transformed into a differential equation with one unknown function $\theta$. Its solution reads

$$
\theta=\phi(z)-\Omega t,
$$

where $\phi$ is an arbitrary function of its variable $z$ while

$$
\Omega=\frac{3 \gamma_{1}+2 \gamma_{2}-3}{6\left(\gamma_{1}+\gamma_{2}\right)}
$$

has the same meaning as in [3].

The imaginary part of (4a) divided by $(G F)$, when substituted with (15) and (16), is equivalent to

$$
\phi_{z}=-2 / 27-s / 3+s^{3}-3 s\left\{\bar{A}_{t t} / \bar{A}+\left[\gamma_{1}+(2 / 3) \gamma_{2}\right] \bar{A}^{2}\right\}
$$

where $\bar{A}=\bar{G} / F$ is the amplitude of $A$ while

$$
s=\Omega-\frac{1}{3}=\frac{\gamma_{1}-3}{6\left(\gamma_{1}+\gamma_{2}\right)} .
$$

The real part of (4a) divided by $(G F)$, when substituted with (15) and (16), is equivalent to

$$
\left[D_{z}+\left(3 s^{2}-1 / 3\right) D_{t}-D_{t}^{3}\right] \bar{G} \cdot F=0 .
$$

Further solution depends on the value of $s$ :

Case 1: $\quad s=0$, i.e. $\Omega=1 / 3, \gamma_{1}=3$.

In this case

$$
\phi=-(2 / 27) z+\phi_{0}, \quad \theta=-(2 / 27) z-(1 / 3) t+\phi_{0}, \quad \phi_{0}=\mathrm{const}
$$

is a special case of (16). Hence the system (4) is no more overdetermined. It follows from Eq. (9) that this case leads to the MKdV equation (with the time $t$ shifted by $z / 3$ ) for the unknown $\bar{A}=\bar{G} / F$ :

$$
\bar{A}_{z}-(1 / 3) \bar{A}_{t}-\left(9+2 \gamma_{2}\right) \bar{A}^{2} \bar{A}_{t}-\bar{A}_{t t t}=0 .
$$

Equation (23) is completely integrable and has well-known multisoliton solutions. This case has already been analyzed in [3].

Case 2: $\quad s \neq 0, \quad$ i.e. $\quad \Omega \neq 1 / 3, \gamma_{1} \neq 3$.

In this case Eq. (18) may be solved for $\bar{A}_{t t}$, which may further be used to reduce the order of (20). Eventually we obtain a first order equation in the form of a conservation law

$$
\bar{A}_{z}+\left[a+\phi_{z} /(3 s)\right] \bar{A}_{t}=0
$$

where 


$$
a=\frac{8}{3} s^{2}+\frac{2}{81 s}-\frac{2}{9}=\frac{2}{27} \frac{3 \gamma_{1}+2 \gamma_{2}-3}{\gamma_{1}-3}\left(\frac{\gamma_{2}+3}{\gamma_{1}+\gamma_{2}}\right)^{2}
$$

This equation may readily be solved by the method of characteristics to give

$$
\bar{A}(z, t)=\bar{A}(0, \tau)=P(\tau),
$$

where $\tau$ is one of the transformed independent variables

$$
\zeta=z, \quad \tau=t-a z-\left[\phi(z)-\phi_{0}\right] /(3 s),
$$

and $\phi_{0}$ is the value of $\phi$ at $z=0$.

Equation (25) is satisfied for an arbitrary function $P$. On the other hand, $P$ should satisfy Eq. (18) which reads in terms of $P$ :

$$
\phi_{z}=-2 / 27-s / 3+s^{3}-3 s\left\{P^{\prime \prime} / P+\left[\gamma_{1}+(2 / 3) \gamma_{2}\right] P^{2}\right\},
$$

where the primes denote derivatives with respect to the variable $\tau$ (the only one on which $P$ depends).

The left hand side does not depend on $\tau$ since $\partial / \partial \tau=\partial / \partial t$ while $\phi$ is independent of $t$. It cannot depend on $\zeta$ either as the right hand side is $\zeta$-independent. Hence $\phi_{z}=$ const, we name this constant $\kappa$. The final solution for $\theta$ reads

$$
\theta(z, t)=\kappa z-\Omega t+\phi_{0}, \quad \phi_{0}=\text { const. }
$$

With constant $\phi_{z}=\kappa$, Eq. (29) has a simple solution in terms of elliptic functions of the variable $\tau$. First (29) may be integrated once to give

$$
P^{\prime 2}=E-b P^{2}-(1 / 2) \gamma_{3} P^{4},
$$

where

$$
b=3 s^{2}-\kappa-1 / 3=3 \Omega^{2}-2 \Omega-\kappa, \quad \gamma_{3}=\gamma_{1}+(2 / 3) \gamma_{2},
$$

while $E$ is the integration constant.

In a way analogous to the NLS we obtain two basic classes of nontrivial finite solutions. Let $\Delta=b^{2}+2 E \gamma_{3}$. Then

Class 1: $\quad b>0, \quad E>0, \quad \gamma_{3}<0, \quad \Delta>0$.

The solution of Eq. (31) reads

$$
P(\tau)= \pm \sqrt{\frac{b-\sqrt{\Delta}}{\left|\gamma_{3}\right|}} \mathrm{sn}\left[\frac{b+\sqrt{\Delta}}{2}\left(\tau-\tau_{0}\right) ; \frac{b-\sqrt{\Delta}}{\sqrt{2 E\left|\gamma_{3}\right|}}\right],
$$

where $\tau_{0}$ is the last integration constant while the function sn is the Jacobi sinus, whose second argument is its modulus. In the limit $\Delta \rightarrow 0$ the modulus tends to 1 and the solution becomes

$$
P(\tau)= \pm \sqrt{b /\left|\gamma_{3}\right|} \tanh \left[\sqrt{b / 2}\left(\tau-\tau_{0}\right)\right]
$$

which describes the well-known optical dark soliton.

Class 2: $\quad b<0, \quad E<0, \quad \gamma_{3}>0, \quad \Delta>0$.

Then

$$
P(\tau)= \pm\left[\frac{p_{1}+p_{2}}{2} \operatorname{dn}(T ; m)+\frac{p_{1}-p_{2}}{2} \operatorname{cn}(T ; m)\right],
$$

where $\mathrm{dn}$ and $\mathrm{cn}$ are the Jacobi elliptic functions, $p_{1}$ and $p_{2}$ are the positive zeros 
of the right hand side of (31)

$$
p_{1}=\sqrt{(|b|+\sqrt{\Delta}) / \gamma_{3}}, \quad p_{2}=\sqrt{(|b|-\sqrt{\Delta}) / \gamma_{3}},
$$

$T$ is rescaled $\tau$ :

$$
T=\left(\gamma_{3} / \sqrt{2}\right)\left(p_{1}+p_{2}\right)\left(\tau-\tau_{0}\right)
$$

while the common modulus of both elliptic functions

$$
m=\left(p_{1}-p_{2}\right) /\left(p_{1}+p_{2}\right) .
$$

In the limit $E \rightarrow 0$ also $p_{2} \rightarrow 0$ and the modulus tends to 1 . Then the solution becomes

$$
P(\tau)=\sqrt{2 b /\left|\gamma_{3}\right|} \operatorname{sech}\left[\sqrt{b}\left(\tau-\tau_{0}\right)\right],
$$

which describes the well-known optical bright soliton.

\section{Conclusion}

We have analyzed the solutions of the HNLS/HONSE equation which may be derived from the Hirota bilinear system (4), proposed in [3]. For the previously handled cases, $\gamma_{1}=3$, the system either reduces to the integrable Hirota equation $[10,3]$ when $\gamma_{2}=-3$ or has multisoliton solutions of the form $A(z, t)=\bar{A}(z, t) \exp \left[-(2 / 27) z-(1 / 3) t+\phi_{0}\right]$, where $\bar{A}(z, t)$ are the respective multisoliton solutions of the MKdV equation (23), for other values of $\gamma_{2}$. In the present paper we have solved the system (4) also for all other cases. In those cases the general solution has the form

$$
A(z, t)=P(\tau) \exp \left[\mathrm{i}\left(\kappa z-\Omega t+\phi_{0}\right)\right]
$$

where $\tau$, given by (28), may be interpreted as the time shifted by the value which the wave needs to get from its initial to its actual position; $P$ is given in terms of elliptic or hyperbolic functions $(34,35,37,41), \kappa$ and $\phi_{0}$ are arbitrary constants and $\Omega$ is given by (17).

This completes the solutions of (4) for the unknown $A(z, t)=G(z, t) / F(z, t)$. The functions $G, F$ can easily be obtained from $\bar{A}$ by solving Eq. (9), which may also be written as

$$
(\ln F)_{t t}=(1 / 2)\left[\gamma_{1}+(2 / 3) \gamma_{2}\right] \bar{A}^{2} .
$$

Having $F$ we immediately get $G=F A$. However, it is the envelope $A$ which is physically relevant whereas $F$ and $G$ themselves are of little interest.

As we see, apart from the case $\gamma_{1}=3$, the only solution of the system (4) is a single solitary wave like in $(35,41)$ or a periodic wave train $(34,37)$. On one hand, it is a natural result: the Painlevé analysis selects this value of $\gamma_{1}$ as the only one for which the system is integrable $[2,3,9]$. On the other hand, the limited shape of the solution may be a result of the overdetermination of the original bilinear system (4). Obtaining the proper bilinear system free of this drawback should be the primary goal of further work. 


\section{References}

[1] V.E. Zakharov, A.B. Shabat, Zh. Eksp. Teor. Fiz. 61, 118 (1971); Sov. Phys. JETP 34, 62 (1972).

[2] S.Y. Sakovich, J. Phys. Soc. Jpn. 66, 2527 (1997).

[3] M. Gedalin, T.C. Scott, Y.B. Band, Phys. Rev. Lett. 78, 448 (1997).

[4] N. Sasa, J. Satsuma, J. Phys. Soc. Jpn. 60, 409 (1990).

[5] K. Porsezian, K. Nakkeran, Phys. Rev. Lett. 76, 3995 (1996).

[6] J. Hietarinta, in: Integrability of Nonlinear Systems, Eds. Y. Kosman-Schwarzbach, B. Grammaticos, K.M. Tamizhmani, Lecture Notes in Physics, Vol. 495, Springer, Berlin 1997, p. 95, Chs. 3.2, 3.3.

[7] R. Hirota, Phys. Rev. Lett. 27, 1192 (1971).

[8] R. Hirota, in: Solitons, Eds. R.K. Bullough, P.J. Caudrey, Springer, Berlin 1980, p. 157.

[9] V. Cao Long, P.P. Goldstein, S. Vu Ngoc, communication at 30th National Annual Meeting on Theoretical Physics, Hué (Vietnam) 2005; Communications in Physics 2006 suppl., 140 (2006).

[10] R. Hirota, J. Math. Phys. 14, 805 (1973).

[11] V. Cao Long, P.P. Goldstein, S. Vu Ngoc, Acta Phys. Pol. A 106, 843 (2004).

[12] V. Cao Long, P.P. Goldstein, M. Trippenbach, Acta Phys. Pol. A 105, 437 (2004). 